The Journal of Experimental Biology 210, 1064-1074

Published by The Company of Biologists 2007

doi: $10.1242 /$ jeb. 02724

\title{
Characterization of very-low density lipoprotein particle diameter dynamics in relation to egg production in a passerine bird
}

\author{
Katrina G. Salvante ${ }^{1, *}$, Gina Lin $^{2}$, Rosemary L. Walzem ${ }^{2}$ and Tony D. Williams ${ }^{1}$ \\ ${ }^{1}$ Department of Biological Sciences, Simon Fraser University, 8888 University Drive, Burnaby, British Columbia, \\ V5A 1S6, Canada and ${ }^{2}$ Poultry Science Department, Texas A\&M University, College Station, TX 77843, USA \\ *Author for correspondence at present address: Department of Biology, Coker Hall, CB 3280, University of North Carolina-Chapel Hill, \\ Chapel Hill, NC 27599-3280, USA (e-mail: ksalvante@unc.edu)
}

Accepted 16 January 2007

\begin{abstract}
Summary
During avian egg production, oestrogen mediates marked increases in hepatic lipid production and changes in the diameter of assembled very-low density lipoprotein (VLDL). A nearly complete shift from generic VLDL ( $\sim 70 \mathrm{~nm}$ in diameter), which transports lipids to peripheral tissues, to yolk-targeted VLDL (VLDLy) $(\sim 30 \mathrm{~nm})$, which supplies the yolk with energy-rich lipid, has been observed in the plasma of laying domestic fowl. We validated an established dynamic laser scattering technique for a passerine songbird Taeniopygia guttata, the zebra finch, to characterize the dynamics of VLDL particle diameter distribution in relation to egg production. We predicted that non-gallinaceous avian species that have not been selected for maximum egg production would exhibit less dramatic shifts in lipid metabolism during egg production. As predicted, there was considerable overlap between the VLDL particle diameter distributions of laying and non-

particles. As a result, laying zebra finches, in comparison, had diameter distributions that were shifted towards larger VLDL particles. Nevertheless, laying zebra finches, like laying chickens, had larger proportions of particles within proposed VLDLy particle diameter ranges than non-laying zebra finches (e.g. sVLDLy: 50\% vs 37\%). Furthermore, zebra finches and chickens had similar modal $(29.7 \mathrm{~nm}$ in both species) and median $(32.7 \mathrm{~nm} v \mathrm{~s}$ $29.6 \mathrm{~nm})$ VLDL particle diameters during egg production. Therefore, although zebra finches and chickens exhibited opposing directional shifts in VLDL particle diameter distribution during egg production, the modifications to VLDL particle structure in both species resulted in the realization of a common goal, i.e. to produce and maintain a large proportion of small VLDL particles of specific diameters that are capable of being incorporated into newly forming egg yolks.
\end{abstract} laying zebra finches. But unexpectedly, non-laying zebra finches had VLDL diameter distributions that peaked at small particles and had relatively few large VLDL
Key words: VLDL, yolk-targeted VLDL, reproduction, VLDL particle diameter, zebra finch.

\section{Introduction}

During avian egg production estrogens stimulate the liver to produce the egg-yolk precursors, yolk-targeted, very-low density lipoprotein (VLDLy) and vitellogenin (VTG), which provide embryos with the energy and nutrients required for growth and development (Gruber, 1972; Bergink et al., 1974; Deeley et al., 1975; Neilson and Simpson, 1973; Chan et al., 1976; Wallace, 1985; Walzem, 1996; Williams, 1998). As a result of increased total hepatic lipid production, plasma lipid concentrations increase from about $3 \mathrm{mg}$ neutral lipid $\mathrm{ml}^{-1}$ plasma in non-laying turkeys (Meleagris gallopavo) to $21 \mathrm{mg}$ in laying turkeys (Bacon et al., 1974). Similarly, plasma triacylglyceride concentration increased from $0.5-1.5 \mu \mathrm{mol} \mathrm{ml}^{-1}$ plasma in non-laying chickens (Gallus gallus domesticus) to $20-50 \mu \mathrm{mol} \mathrm{m}^{-1}$ plasma in laying chickens (Griffin and Hermier, 1988). Furthermore, data for egg-laying chickens, turkeys and quail (Coturnix coturnix) show that there is an oestrogen-dependent shift in VLDL synthesis from the production of generic VLDL, which ranges in size from 30 to $>200 \mathrm{~nm}$, to smaller, yolk-targeted VLDL, which ranges in diameter from 15 to $55 \mathrm{~nm}$ in domestic fowl (Chapman, 1980; Griffin, 1981; Walzem et al., 1994; Walzem, 1996; Speake et al., 1998; Chen et al., 1999; Walzem et al., 1999). Furthermore, whereas generic VLDL has at least six associated apolipoproteins (including apoA-I, apoB and apoC), VLDLy has only two associated apolipoproteins, apoB and apoVLDL-II, of which the latter is thought to be responsible for the decrease in VLDLy diameter (Chan et al., 1976; Kudzma et al., 1979; Griffin, 1981; Dashti et al., 1983; Lin et al., 1986; Schneider et al., 1990; Speake et al., 1998; Walzem et al., 1999). Consequently, the presence of circulating VLDLy in egg-producing females represents a dramatic shift in lipid 
metabolism associated with changes in the composition and structure of VLDL.

The structural changes to circulating VLDL particles directly influence their in vivo function during egg production (Walzem, 1996). Whereas the role of generic VLDL is to transport triacylglycerides throughout the body for tissue utilization or storage in adipose tissue, the function of VLDLy is to deliver triacylglycerides to the oocyte, where they will be used as the energy source for the developing embryo (Walzem, 1996). The smaller diameter of VLDLy is thought to be critical for enabling the particles to pass through the pores in the granulosa basal lamina of the ovary, allowing them access to the developing ovarian follicles (Griffin and Perry, 1985). In addition, apoVLDL-II also acts as an inhibitor of lipoprotein lipase (LPL), probably by limiting access to the water needed for triacylglycerol hydrolysis (Boyle-Roden and Walzem, 2005). The resistance of VLDLy particles to hydrolysis by extra-ovarian tissues preserves the triacylglycerol-rich VLDLy for uptake by the developing ovarian follicles (Walzem, 1996). Cross-injection studies on turkeys and chickens using labelled generic VLDL isolated from immature turkeys and labelled VLDLy from laying turkeys and chickens confirm that immature and laying birds utilize generic VLDL and VLDLy differently; a greater proportion of generic VLDL was deposited into tissues, whereas more VLDLy was incorporated into ovarian follicles (Bacon et al., 1978; Bacon, 1981). In vivo studies in laying domestic fowl have detected only low circulating levels of intermediate-density and low-density lipoproteins, both by-products of the hydrolysis of VLDL by LPL (Hermier et al., 1989; Walzem et al., 1994; Walzem, 1996), providing further evidence for the increased in vivo resistance of VLDLy to LPL hydrolysis.

Despite the LPL-resistance of VLDLy, chickens and turkeys are able to incorporate radiolabelled VLDLy from laying females into non-ovarian tissues, presumably to metabolize them to meet their energetic needs (Bacon et al., 1978; Bacon, 1981). Griffin and Hermier (Griffin and Hermier, 1988) noted that some $10 \%$ of VLDLy triacylglycerol can be hydrolyzed by LPL; given the high plasma concentrations of VLDLy in laying domestic fowl, this partial hydrolysis may be sufficient to meet the female's own energetic requirements during laying. Others (Chen et al., 1999; Walzem et al., 1999) have proposed that laying domestic fowl could also potentially meet their energetic requirements by metabolizing small amounts of generic VLDL that are synthesized by the avian kidney (Blue et al., 1980; Tarugi et al., 1998).

Chickens have been the target of strong artificial selection for prolonged and consistent egg production, and can maintain high rates of egg production for over a year (Etches, 1996). It is not known whether non-domesticated, non-gallinaceous avian species, which have not been selected for maximum egg production, exhibit such dramatic shifts in lipid metabolism during egg production. Passerine birds have been shown to experience marked increases in the concentration of circulating triacylglycerol during egg production (Christians and Williams, 1999; Challenger et al., 2001). However, the assay used in these studies measured triacylglycerides associated with both generic and yolk-targeted VLDL and was not able to distinguish between the two forms of the lipoprotein. A dynamic laser scattering technique was used to assess VLDL particle diameter distribution in domestic fowl (Walzem et al., 1994; Walzem et al., 1999). This method provides a frequency distribution of VLDL particle diameters (in $\mathrm{nm}$ ), which has been shown to vary in relation to egg production (Walzem et al., 1994; Walzem, 1996; Walzem et al., 1999; Peebles et al., 2004). At present there is a paucity of data on VLDL particle diameter distributions for nondomesticated species. In this paper we describe a modification of the dynamic laser scattering technique for use in a passerine songbird, the zebra finch. We used this technique to (1) characterize VLDL particle diameter distributions in nonlaying and egg-producing female zebra finches, (2) estimate the diameter range of VLDL particles that are available for deposition into the developing eggs of laying zebra finches, and (3) compare VLDLy dynamics during egg production in zebra finches and chickens. Non-domesticated birds have not been strongly selected for egg production and generally experience much more variable environmental conditions during egg production, including fluctuations in food availability and low ambient temperature. We, therefore, predicted that laying female passerines should experience greater selection pressures to be able to maintain production of larger, potentially generic, VLDL during egg production in order to meet their unpredictable energetic needs.

\section{Materials and methods \\ Animal husbandry}

Zebra finches (Taeniopygia guttata Vieillot) were housed in the Simon Fraser University Animal Care Facility under controlled environmental conditions (temperature $19-23^{\circ} \mathrm{C}$, humidity $35-55 \%$, constant photoperiod of $14 \mathrm{~h}: 10 \mathrm{~h} \mathrm{~L}: \mathrm{D}$; lights on at 07:00 h). All birds received a mixed seed diet (Panicum and white millet, 50:50; approximately $12.0 \%$ protein, $4.7 \%$ lipid; Jameson's Pet Food, Vancouver, BC, Canada and Just for Birds, Surrey, BC, Canada), water, grit and cuttlefish bone (calcium) ad libitum. Birds also received a multivitamin supplement in the drinking water once per week. When not paired for breeding, the birds were housed in samesex cages, but were not visually or acoustically isolated from the opposite sex. All experiments and animal husbandry were carried out under a Simon Fraser University Animal Care Committee permit (no. 692B-94) following the guidelines of the Canadian Committee on Animal Care.

Single comb white leghorn chickens of the W-36 strain (HyLine International, Bryan, Texas, USA) were individually housed in light-supplemented, fan-ventilated, open-sided houses at the University of California, Davis, USA. Chickens were given ad libitum access to water and a corn-soy diet formulated to meet the National Research Council for Poultry's (NRC) requirements for laying hens (15\% protein, $<5 \%$ lipid, $2900 \mathrm{kcal} \mathrm{kg}^{-1}$ ) National Research Council Subcommittee on 
Poultry Nutrition, 1994), and were provided with $15 \mathrm{~h}$ of light per day. Ambient house temperature varied from $7-29^{\circ} \mathrm{C}$. All animal husbandry and experimental procedures were conducted in accordance with a protocol approved by the Animal Use and Care Committee of the University of California, Davis, USA.

\section{Zebra finch breeding and blood sampling}

Male $(N=36)$ and female $(N=36)$ zebra finches were weighed $( \pm 0.1 \mathrm{~g})$ at the time of pairing, and tarsus and bill measurements $( \pm 0.1 \mathrm{~mm})$ were taken. Breeding pairs were housed individually in cages $(61 \times 46 \times 41 \mathrm{~cm}$; length $\times$ width $\times$ height $)$ equipped with an external nest box $(15 \times 14.5 \times 20 \mathrm{~cm})$ and were provided with an egg-food supplement $(6 \mathrm{~g}$ of a mixture of 62-65 g hard-boiled egg, $13 \mathrm{~g}$ cornmeal, $13 \mathrm{~g}$ bread crumbs; $30.2 \%$ protein and $13.0 \%$ lipid by dry mass) daily between pairing and clutch completion in addition to the normal seed diet (see Williams, 1996). Data on laying interval (days from pairing to initiation of laying) and egg and clutch size were obtained by checking the nest boxes daily between 09:00 $\mathrm{h}$ and 11:00 h. All new eggs were weighed $( \pm 0.001 \mathrm{~g})$ and numbered on the day they were laid. Clutches were considered complete if no new eggs were laid over 2 days. At this time the female was weighed, and the pair was returned to same-sex, nonbreeding cages.

Laying females were weighed and blood samples taken (200 $\mu \mathrm{l}$ from the brachial vein) on the day their first eggs were laid (laying sample). Randomly chosen female zebra finches $(N=27)$ from the same-sex cages were also weighed and blood samples taken (non-laying sample). All blood samples were collected between 09:00 h and 11:30 h into heparinized capillary tubes, and then expelled into EDTA-coated microcentrifuge tubes containing $0.5 \mathrm{~mol}^{-1}$ disodium-EDTA ( $3 \mu \mathrm{l}$; Sigma-Aldrich Canada, Oakville, ON, Canada) and centrifuged at $2200 \mathrm{~g}$ for $10 \mathrm{~min}$ in a Baxter Canlab Biofuge 13. A sub-sample $(5 \mu \mathrm{l})$ of each plasma sample was frozen $\left(-20^{\circ} \mathrm{C}\right)$ for triacylglyceride analysis, and the remainder of each plasma sample was placed into an EDTA-coated microcentrifuge tube containing $0.5 \mathrm{~mol} \mathrm{l}^{-1}$ disodium-EDTA $(5 \mu \mathrm{l})$ for VLDL particle diameter distribution analysis. Sodium azide (1\% w:v; Sigma-Aldrich Canada) was added to each EDTA-coated tube to prevent mould formation (0.01 $\mu \mathrm{l} \mu \mathrm{l}^{-1}$ plasma), and the plasma samples were refrigerated $\left(4^{\circ} \mathrm{C}\right)$ pending analysis of VLDL particle diameter distribution.

\section{Influence of feeding, fasting and egg-food supplementation on VLDL particle diameter distribution in zebra finches}

Two preliminary studies were performed to assess the potentially confounding effects of feeding $v s$ fasting and diet supplementation (i.e. the egg-food supplement given to breeding pairs) on various measures of VLDL particle diameter distribution and circulating triacylglyceride levels. For the fasting experiment, randomly selected laying female zebra finches were blood sampled twice; once while in the 'fed' state, i.e. ad libitum access to seed and egg-food supplement and again while in the 'fasted' state, i.e. 15-16 h without access to food. For the 'fasted' sample, the seed and egg-food supplement containers were removed at 19:00 on the night before blood sample collection. Female zebra finches were weighed and blood sampled between 10:00 $\mathrm{h}$ and 11:00 $\mathrm{h}$ on the days that their second and third eggs were laid (2-egg and 3 -egg stages, respectively). The order in which females were in the fed and fasted states was randomized such that half of the females were in the fasted state at the 2-egg and in the fed state at the 3-egg stage, whereas the reverse was true for the other half of the females. Previous studies on vitellogenin (VTG), the other oestrogen-dependent yolk precursor, have reported comparable levels of plasma VTG levels at the 2- and 3-egg stages (Challenger et al., 2001; Salvante and Williams, 2002). Seed consumption during the $24 \mathrm{~h}$ prior to each blood sample was measured by providing each breeding pair with $30.0 \mathrm{~g}$ of seed on the days the females laid their first and second eggs. Seed was weighed (to the nearest $0.1 \mathrm{~g}$ ) $24 \mathrm{~h}$ later at the 2- and 3-egg stages.

To examine the influence of the high-fat egg-food supplement given to breeding pairs on changes in plasma triacylglyceride levels and VLDL particle diameter distribution during egg production, randomly chosen male zebra finches were weighed and blood samples were taken between 09:00 h and $11: 30 \mathrm{~h}$ on two separate occasions: as non-breeding individuals on the seed-only diet (seed sample), and during breeding on the egg-food supplemented seed diet (supplemented sample) on the day that their female breeding partners laid their first eggs.

\section{Chicken blood sampling}

Blood samples for VLDL particle diameter distribution analysis were collected from two groups of chickens: immature, non-laying females at 17 weeks of age $(N=10)$ and actively laying females at 29 weeks of age $(N=37)$. Sampling of the 29-week-old layers coincided with the peak of laying for the population (i.e. all females were actively laying eggs and the laying rate for the population was at its peak: 0.9 eggs laid per day). Blood samples were taken from the brachial vein between 09:00 and 11:00 into EDTA-coated Vacutainer tubes (BD Diagnostics, Franklin Lakes, NJ, USA). Plasma samples were isolated by centrifugation at $2200 \mathrm{~g}$ and were refrigerated $\left(4^{\circ} \mathrm{C}\right)$ pending VLDL particle diameter distribution analysis.

\section{Triacylglyceride assay}

Circulating concentrations of triacylglyceride in zebra finches were measured enzymatically as an index of total plasma VLDL (i.e. generic VLDL and VLDLy; Triglyceride E kit; Wako Chemicals, Richmond, BC, Canada; Serum Triglyceride Determination Kit, Sigma-Aldrich Canada) using the method developed for domestic fowl (Mitchell and Carlisle, 1991) and validated for passerines (Williams and Christians, 1997; Williams and Martyniuk, 2000; Challenger et al., 2001). Intra- and inter-assay coefficients of variation were $1.85 \%$ ( $N=6$ replicates) and $4.79 \%$ ( $N=13$ assay plates), respectively, using a 19-week hen plasma pool. All assays were run using 
96-well microplates and were measured at $540 \mathrm{~nm}$ using a Biotek 340i microplate reader.

\section{VLDL particle diameter distribution assay}

Whole plasma contains a variety of different lipoprotein classes, e.g. VLDL, low density lipoprotein (LDL), high density lipoprotein (HDL). Therefore, plasma VLDL was isolated as the $d<1.020 \mathrm{~g} \mathrm{ml}^{-1}$ fraction of plasma from zebra finches and chickens. The volume of each zebra finch plasma sample (approximately $100 \mu \mathrm{l}$ ) was measured and transferred into Beckman Ultra-Clear ultracentrifuge tubes $(13 \times 64 \mathrm{~mm}$, \#344088; Beckman Coulter, Fullerton, CA, USA), and $\mathrm{NaCl}$ density solution $(d=1.0063$; equivalent salt density of undiluted plasma) was added until a final volume of $1 \mathrm{ml}$ was reached. Alternatively, a sub-sample $(1 \mathrm{ml})$ from each chicken plasma sample was transferred into ultracentrifuge tubes. $\mathrm{NaCl}-\mathrm{NaBr}$ density solution ( $5 \mathrm{ml} ; d=1.0255)$ was then added to each tube. A blank sample was prepared by combining $\mathrm{NaCl}$ density solution ( $1 \mathrm{ml} ; d=1.0063)$ with $\mathrm{NaCl}-\mathrm{NaBr}$ density solution ( $5 \mathrm{ml} ; d=1.0255)$ in an ultracentrifuge tube. The samples were loaded into a Beckman 50.4 fixed-angle rotor and centrifuged at $148600 \mathrm{~g}$ for $18 \mathrm{~h}$ at $14^{\circ} \mathrm{C}$ in a Beckman L8-70M ultracentrifuge (Beckman Coulter, Fullerton, CA, USA). Following centrifugation, the supernatant containing the VLDL portion of the plasma was isolated from each tube by aspiration with a narrow-bore pipette and refrigerated (at $4^{\circ} \mathrm{C}$ ) until analysis for VLDL particle diameter distribution. VLDL particle diameter distribution was measured by dynamic laser light scattering using a UPA 250 and 7.02 analysis software (Microtrac, Montgomery, PA, USA) (Walzem et al., 1994; Véniant et al., 2000). This technique utilized the Doppler effect as the basis for diameter distribution determinations by recording light scattering from a directed laser diode as it passed through the lipoprotein particles. The magnitude of Doppler-shifting of light scatter that occurs due to the Brownian motion of the particles was measured as it is proportional to particle velocity, which is in turn a function of particle diameter, fluid temperature and fluid viscosity. As both temperature and viscosity were kept constant, the difference in particle velocity was solely dependent on particle diameter. Sample measurements were made by placement of the flexible probe-tip into the sample and activation of the laser diode $(\lambda=780 \mathrm{~mm}$ laser beam). Light scattering from the lipoprotein particles was recorded for 3 min for the blank solution, and $5 \mathrm{~min}$ in triplicate for each VLDL sample. The probe was washed with distilled water and dried between samples.

\section{Data analysis}

\section{$V L D L$ particle diameter distribution measurements}

To determine the range of VLDL particle diameters involved in egg production in passerine songbirds, we characterized changes in VLDL particle diameter distribution in zebra finches in comparison to chickens by calculating the proportion of VLDL particles that fell within three potential VLDLy particle diameter ranges: (1) a range based on chicken values (hereafter referred to as the cVLDLy range), (2) a range based on the proposed sieving properties of the avian ovary (hereafter referred to as the sVLDLy range), and (3) a range based on zebra finch values (hereafter referred to as the zVLDLy range). Walzem calculated VLDLy particle diameter range for laying chickens, the cVLDLy range, using the regression of the percentage of VLDL particles within each VLDL particle diameter class against subsequent laying rate, the most common measure of reproductive effort used for domestic fowl (Walzem, 1996). Chickens lay continuously for extended periods. Therefore, laying females were repeatedly sampled at various times throughout the laying period, and these repeated measures were incorporated into the VLDLy calculations (Walzem, 1996). Each of the resulting correlation coefficients $(r)$ was presented graphically as $y$-values for each particle diameter class. The different VLDL particle diameter classes vary in their ability to support continuous egg production, and the diameter classes with positive relationships (i.e. positive correlation coefficients) with laying rate were assumed to have a better ability to support egg production and were therefore selected to make up the cVLDLy particle diameter range [21.5-51.1 nm for laying chickens; Fig. 1A; fig. 6 in Walzem

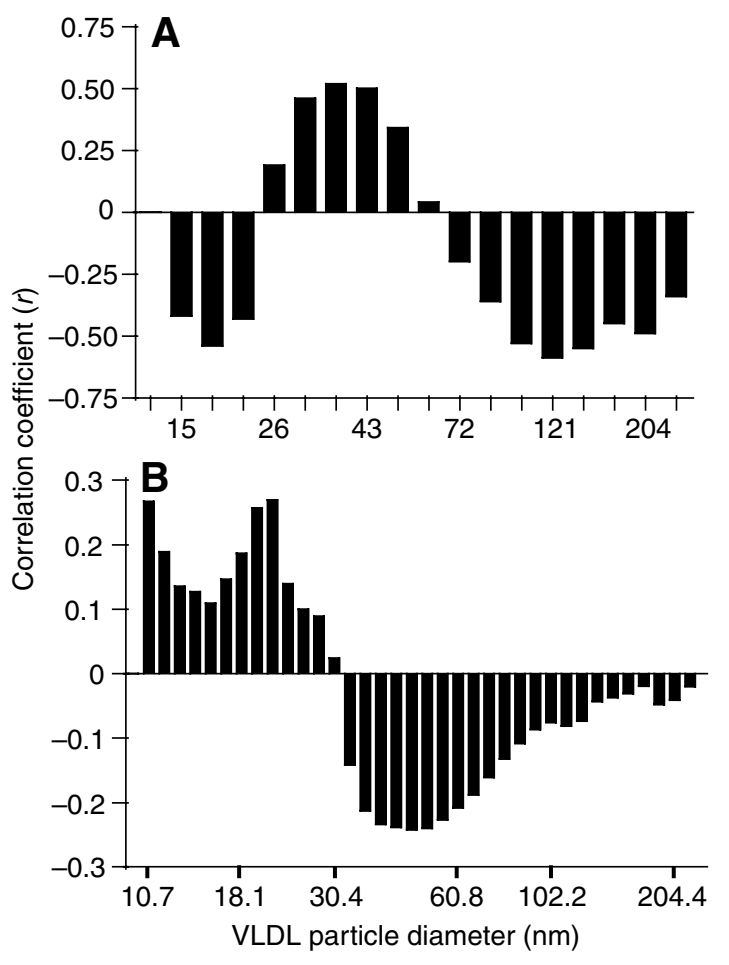

Fig. 1. Histogram plots of correlation coefficients, $r$, for specific particle diameter classes of very-low density lipoprotein (VLDL) isolated from the plasma of (A) laying chickens [fig. 6 from Walzem (Walzem, 1996)], and (B) laying zebra finches. Correlation coefficients were generated from correlations between the proportion of VLDL particles within each diameter class and subsequent egg production (i.e. laying rate in chickens and mean egg mass in zebra finches). VLDL particle diameter classes with $r>0$ were positively associated with egg production, and were therefore included in estimates of yolk-targeted VLDL (VLDLy) particle diameter range. 
(Walzem, 1996)]. By contrast, the sVLDLy range was based on the observation in domestic fowl that the only VLDL particles observed distal to the granulosa basal lamina of the ovary during yolk formation, and thus able to reach the plasma membrane of the enlarging oocyte of the developing ovarian follicles, ranged from 25 to $44 \mathrm{~nm}$ in diameter (Perry and Gilbert, 1979; Griffin and Perry, 1985; Griffin and Hermier, 1988). These studies suggested that pores in the granulosa basal lamina act as selective sieves, allowing only VLDL particles of certain diameters to filter into the ovary (Perry and Gilbert, 1979; Griffin and Perry, 1985; Griffin and Hermier, 1988). Finally, the zVLDLy range (10.7-30.4 nm) was calculated similarly to the cVLDLy range described above with the following exceptions. Firstly, because zebra finches lay discrete clutches (5-7 eggs), laying females were only blood sampled once, on the day their first eggs were laid. Therefore, only one set of VLDL particle diameter and reproductive output data was used per bird (cf. the repeated measures of VLDL particle diameter and reproductive performance incorporated into the chicken VLDLy analysis because of their continuous laying). Secondly, because laying rate is not generally informative in zebra finches (i.e. there is virtually no variation in laying rate because the majority of females lay one egg per day without skipping a day until the clutch is complete), we used body mass-corrected mean egg mass as a measure of reproductive performance in zebra finches (Fig. 1B). Mean egg mass varies markedly between individual female zebra finches (Williams, 1996; Salvante and Williams, 2002), but is highly repeatable within individual females between laying bouts (Williams, 1996), suggesting that mean egg mass is a distinct phenotypic trait of laying zebra finches. There is also evidence that egg size reflects a female's 'egg laying ability' or 'performance'; females that lay large eggs are more capable of laying extended clutches in response to egg removal than females that lay small eggs (Williams and Miller, 2003). VLDLy particle diameter ranges based on other measures of reproductive performance in zebra finches (e.g. clutch size, clutch mass) were also determined but were not used because they encompassed a majority of the VLDL particle diameter classes (i.e. 30 to $>200 \mathrm{~nm}$ ), making them inconsistent with other potential VLDLy diameter range estimates. Finally, the modal and median particle diameter and the range (i.e. width) of each distribution, in nanometres, and the proportion of very small $(<30 \mathrm{~nm})$ and large $(>51 \mathrm{~nm})$ VLDL particles were also determined.

\section{General statistics}

All statistical analyses were performed using SAS (SAS Institute, 1999). All percentage data (e.g. percentage of VLDL particles within the various VLDLy diameter ranges) were arc-sin transformed prior to analysis, however, nontransformed percentages were used for graphical purposes. Non-normal variables, as assessed by the Shapiro-Wilk test for normality (Zar, 1996), were normalized through $\log _{10}$ transformation (although some non-transformed values were used for graphical purposes). For intra-specific comparisons of the VLDL particle diameter distributions of laying and nonlaying females, $t$-tests were used. When the analyses included variables that were still not normally distributed after logtransformation (e.g. plasma triacylglyceride and VLDL particle diameter distribution range of zebra finches, and modal and median VLDL particle diameter and VLDL particle diameter distribution range of chickens), non-parametric Wilcoxon ranksum tests were performed. The influence of fasting and eggfood supplementation on VLDL particle diameter distribution was assessed using repeated-measures ANOVA or ANCOVA (with female body mass as a covariate). If normality of distribution was achieved following data transformation, then the data were analyzed using a mixed model, repeated measures ANOVA or ANCOVA with fed-fasted state for the fasting study or diet for the egg-food supplementation study as the fixed, repeated factor, and individual bird as a random factor (PROC MIXED) (SAS Institute, 1999). By contrast, variables that were still not normally distributed following data transformation were analyzed using the non-parametric Friedman's test for treatment differences in a randomized complete block design with individual birds as blocks that received both treatments (i.e. fed and fasted states or seed and egg-food supplemented seed diets) in a randomized order (PROC FREQ) (SAS Institute, 1999). All values are given as means \pm s.e.m., all tests are two-tailed, and the overall significance level is $P<0.05$ unless otherwise stated.

\section{Results \\ Influence of feeding, fasting and egg-food supplementation on VLDL particle diameter distribution in zebra finches}

Breeding pairs consumed an average of $37 \%$ less seed while being fasted for part of the day than while having ad libitum access to seed throughout the day $\left(F_{1,13}=119.66, P<0.0001\right.$; Fig. 2A). This decrease in daily seed intake resulted in significant declines in female body mass (by an average of $0.44 \mathrm{~g} ; \quad F_{1,13}=7.21, \quad P<0.025 ; \quad$ Fig. $\left.2 \mathrm{~B}\right) \quad$ and circulating triacylglyceride levels (by an average of $1.6 \mathrm{mg} \mathrm{ml}^{-1}$ plasma, i.e. $14 \% ; F_{1,13}=7.26, P<0.025$; Fig. 2 C) following fasting. By contrast, fasting for $15-16 \mathrm{~h}$ had no effect on the proportion of VLDL particles within the cVLDLy, sVLDLy (Fig. 2D), or zVLDLy ranges, modal (Fig. 2E) and median VLDL particle diameter, VLDL particle diameter distribution range (Fig. 2F) or the proportion of very small $(<30 \mathrm{~nm})$ or large $(>51 \mathrm{~nm})$ VLDL particles in circulation $(P>0.1$ in all cases).

When provided with the egg-food supplemented seed diet during breeding, male zebra finches actually lost an average of $13 \%$ of their body mass compared to when they were maintained on the seed-only diet as non-breeders $\left(F_{1,16}=37.24\right.$, $P<0.0001$; Fig. 3A). By contrast, egg-food supplementation during egg production did not influence the circulating triacylglyceride levels (Fig. 3B), modal (Fig. 3C) or median VLDL particle diameter, VLDL particle diameter distribution range (Fig. 3D), or the proportion of very small $(<30 \mathrm{~nm})$ or large $(>51 \mathrm{~nm})$ VLDL particles in circulation in breeding males $(P>0.05$ in all cases $)$. 

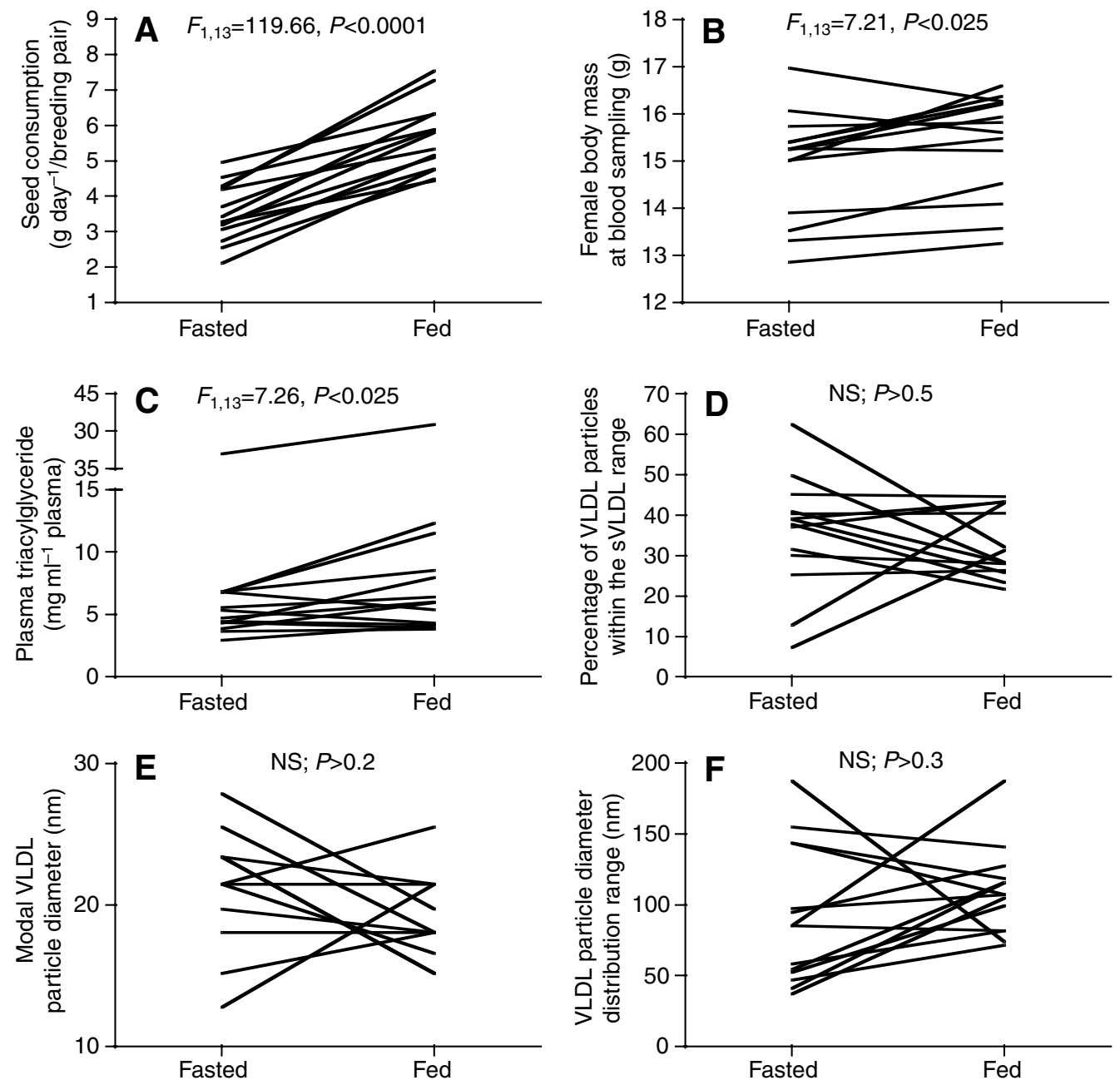

Fig. 2. Influence of fasting on (A) daily seed consumption of breeding pairs of zebra finches, and on (B) body mass, (C) plasma triacylglyceride levels, (D) the proportion of very-low density lipoprotein (VLDL) particles available for use by the developing ovarian follicles as defined by the proposed selective sieving properties of the ovary, i.e. the proportion of particles that fell within the sVLDLy diameter range (25-44 nm), (E) modal VLDL particle diameter, and (F) VLDL particle diameter distribution range of laying female zebra finches.

Plasma triacylglyceride and VLDL particle diameter distribution in non-laying and egg-laying zebra finches

Whereas laying and non-laying female zebra finches did not differ in body mass at the time of blood sampling $(P>0.2$; Table 1), laying zebra finches had higher plasma triacylglyceride levels than non-laying females (Wilcoxon rank-sum test: $Z=-4.008, P<0.0005$; Table 1). By contrast to the results from laying chickens (see Introduction), non-laying zebra finches had VLDL particle diameter distributions (Fig. 4A) that were narrow (177 nm wide, cf. $233 \mathrm{~nm}$ in laying zebra finches; $Z=-1.980, P<0.05$; Table 1$)$ and peaked at very small particle diameters (over 55\% of particles had diameters smaller than $30 \mathrm{~nm}$, cf. less than $30 \%$ in laying zebra finches; $t=5.867$, d.f. $=61.0, P>0.0001$; Table 1$)$ and contained few large particles (less than $10 \%$ of particles had diameters larger than $51 \mathrm{~nm}$, cf. almost $20 \%$ in laying zebra finches; $t=3.947$, d.f. $=59.2, P<0.0005$; Table 1). Furthermore, non-laying zebra finches also had smaller modal $(t=4.405$, d.f. $=61.0, P<0.0001)$ and median $(t=5.332$, d.f. $=61.0, P<0.0001)$ VLDL particle diameters than laying females (Table 1). Therefore, in comparison, laying zebra finches had VLDL particle diameter distributions that were shifted towards larger VLDL particle diameters compared to non-laying females (Fig. 4A). Although there was considerable overlap between the diameter distributions of laying and non-laying zebra finches, laying females still had greater proportions of VLDL particles within the cVLDLy $(t=2.866$, d.f. $=30.2, P<0.05)$ and sVLDLy ranges $(t=3.058$, d.f. $=31.0, P<0.005$; grey shading in Fig. 4A) than non-laying females (Table 1). However, laying zebra finches had fewer VLDL particles within the zVLDLy range than nonlaying birds $(t=4.581$, d.f. $=61.0, P<0.0001$; Table 1$)$.

\section{$V L D L$ particle diameter distribution in non-laying and egg- laying chickens}

Non-laying chickens were consistently different from laying chickens in all measures of VLDL particle diameter 

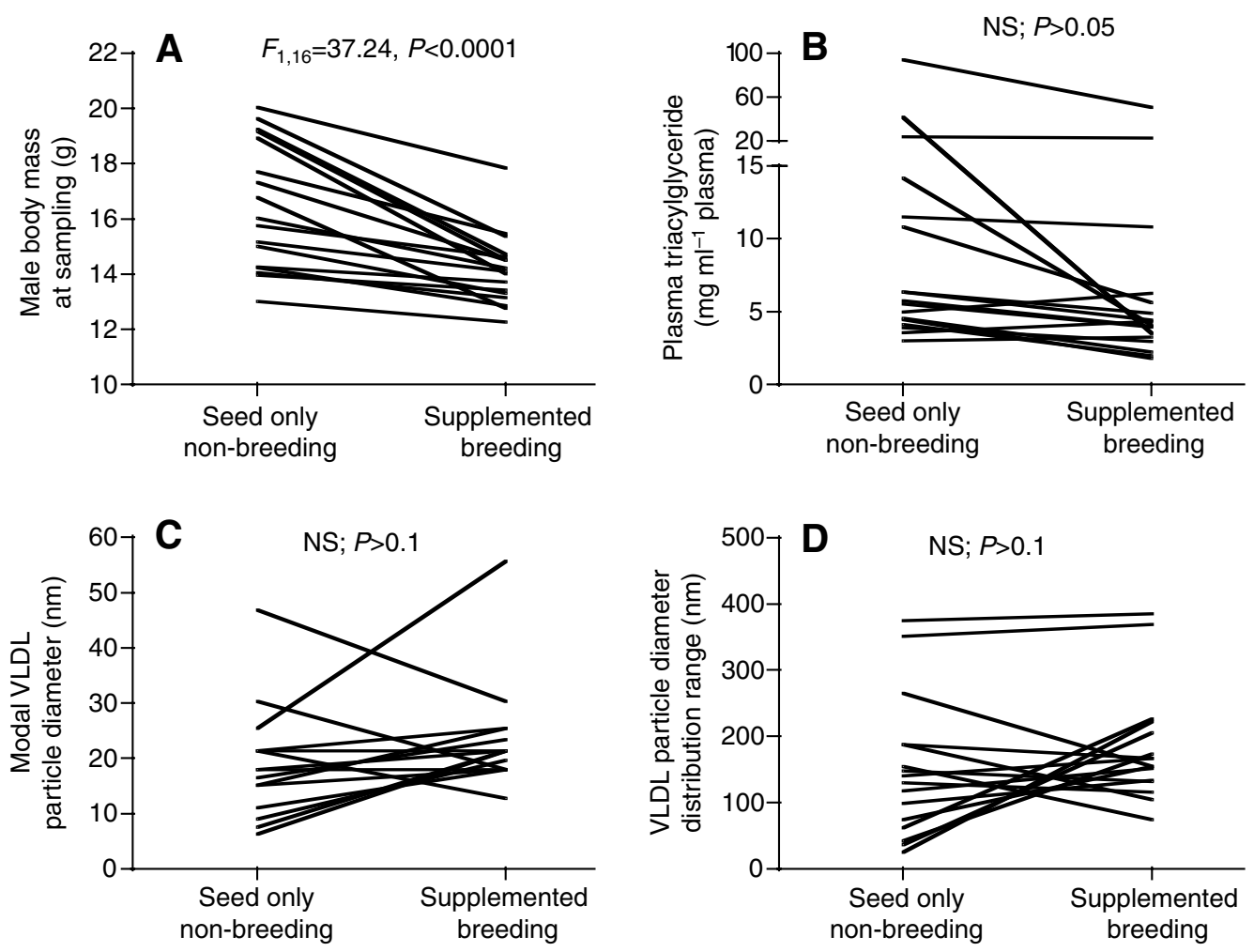

Fig. 3. The effects of egg-food supplementation and reproductive activity on (A) body mass, (B) plasma triacylglyceride levels, (C) modal verylow density lipoprotein (VLDL) particle diameter and (D) VLDL particle diameter distribution range of male zebra finches.

distribution. On average, VLDL particle diameter distributions of laying chickens at 29-weeks of age were narrow, and peaked at small particle diameters (Fig. 4B), while non-laying chickens possessed wider, less peaked distributions (Fig. 4B) (range: $Z=4.816, P<0.0001$; Table 1). Laying chickens had a larger proportion of VLDL particles that fell within the cVLDLy range ( $t=9.542$, d.f. $=10.3, P<0.0001$; grey shading in Fig. 4B) and sVLDLy range $(t=8.909$, d.f. $=10.3, P<0.0001)$ than nonlaying chickens (Table 1). Unlike in zebra finches, non-laying chickens had larger modal $(Z=3.818, P<0.0001)$ and median $(Z=4.797, P<0.0001)$ particle diameters, fewer smaller VLDL particles $(<30 \mathrm{~nm}$ in diameter; $t=25.990$, d.f. $=43.2, P<0.0001)$ and more large VLDL particles ( $>51 \mathrm{~nm}$ in diameter; $t=11.314$, d.f. $=9.1, P<0.0001)$ than laying chickens (Table 1$)$.

Table 1. Total plasma triacylglyceride and measurements of very-low density lipoprotein particle diameter distribution for nonlaying and laying female zebra finches and chickens

\begin{tabular}{|c|c|c|c|c|}
\hline \multirow[b]{2}{*}{ Trait } & \multicolumn{2}{|c|}{ Zebra finches } & \multicolumn{2}{|c|}{ Chickens } \\
\hline & Non-laying & Laying & Non-laying & Laying \\
\hline Mass at blood sampling (g) & $15.83 \pm 0.31(27)$ & $16.11 \pm 0.21(36)$ & - & - \\
\hline Particles within the zVLDLy range $(10.7-30.4 \mathrm{~nm})(\%)$ & $59.33 \pm 3.48(27)$ & $39.57 \pm 2.67(36) * * * *$ & - & - \\
\hline Particles within the cVLDLy range $(21.5-51.1 \mathrm{~nm})(\%)$ & $50.77 \pm 4.27(27)$ & $63.47 \pm 1.20(36)^{*}$ & $25.14 \pm 4.51(10)$ & $69.66 \pm 1.21(37) * * * *$ \\
\hline Particles within the sVLDLy range $(25-44 \mathrm{~nm})(\%)$ & $37.96 \pm 3.74(27)$ & $49.95 \pm 1.16(36)^{* *}$ & $18.89 \pm 4.54(10)$ & $60.77 \pm 1.23(37) * * * *$ \\
\hline VLDL particle diameter distribution range (nm) & $177.1 \pm 17.0(27)$ & $232.9 \pm 19.0(36)^{*}$ & $329.5 \pm 15.4(10)$ & $76.1 \pm 6.7(37)^{* * * *}$ \\
\hline Particles with diameters $<30 \mathrm{~nm}(\%)$ & $57.20 \pm 4.17(27)$ & $27.81 \pm 3.01(36)^{* * * *}$ & $0.51 \pm 0.51(10)$ & $38.64 \pm 1.38(37) * * * *$ \\
\hline Particles with diameters $>51 \mathrm{~nm}(\%)$ & $9.65 \pm 1.33(27)$ & $18.64 \pm 1.85(36)^{* * *}$ & $59.09 \pm 4.39(10)$ & $9.19 \pm 0.34(37) * * * *$ \\
\hline
\end{tabular}

VLDL, very-low density lipoprotein; zVLDLy and cVLDLy, zebra finch and chicken yolk-targeted VLDL, respectively; sVLDLy, VLDLy within the range of the 'sieving' properties of the avian ovary.

Values are means \pm s.e.m., with sample size in parentheses. All percentages are arc-sine transformed.

$* P<0.05, * * P<0.005, * * * P<0.0005$ and $* * * * P<0.0001$ for intra-specific comparisons. 


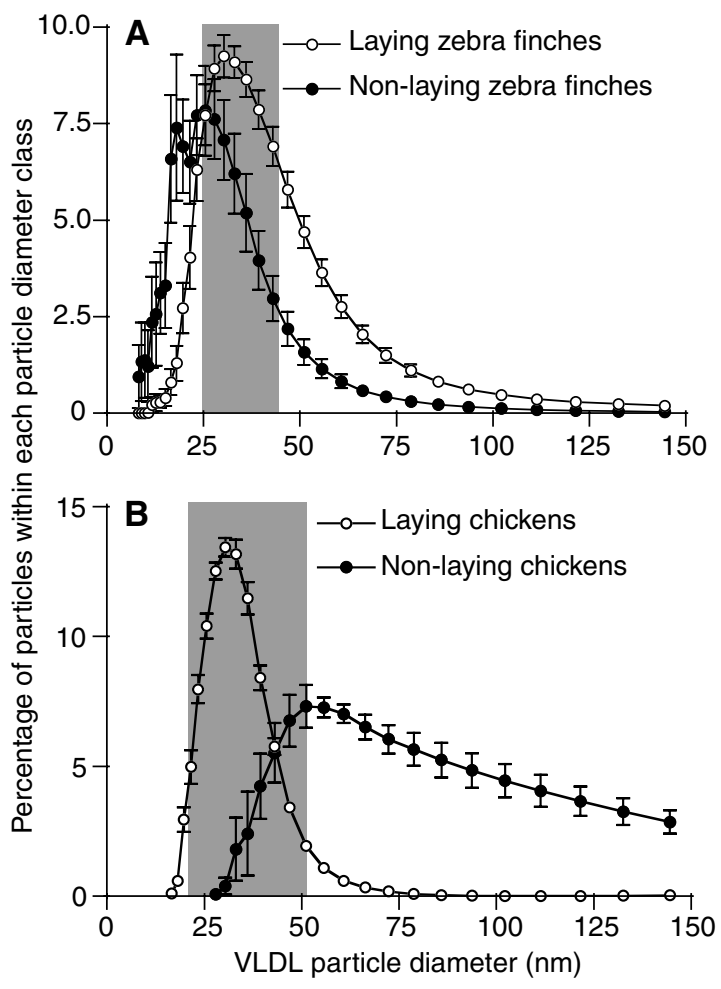

Fig. 4. Very-low density lipoprotein (VLDL) particle diameter distributions of laying and non-laying female (A) zebra finches and (B) chickens. Grey shading indicate estimates of yolk-targeted VLDL (VLDLy) particle diameter range based on (in A) the proposed sieving properties of the ovary limiting access of VLDL particles to the developing ovarian follicles (sVLDLy range: $25-44 \mathrm{~nm}$ ) or (in B) the idea that there is a positive relationship between VLDL particle diameter classes and laying rate in chickens that are better able to support continuous egg production (cVLDLy range: 21.5-51.1).

\section{Discussion}

Influence of feeding, fasting and egg-food supplementation on $V L D L$ particle diameter distribution in zebra finches

Although all of the chickens and zebra finches used in this study had ad libitum access to high quality food, the diet that laying zebra finches were provided with had a higher lipid content (4.7\% lipid from seed and 13\% from egg-food supplement) than the non-laying zebra finches ( $4.7 \%$ lipid from seed only diet) and the laying chicken diets $(<5 \%)$. To confirm that differences observed in VLDL particle diameter distribution parameters between species and between reproductive stages in zebra finches were independent of differences in lipid intake or diet quality, we examined the influence of fasting and egg-food supplementation on various measures of VLDL particle diameter distribution in zebra finches. The various measures of VLDL particle diameter distribution did not differ with respect to the fed-fasted status of laying zebra finches. Likewise, male zebra finches exhibited comparable VLDL particle diameter distributions when maintained on the seed-only diet as non-breeders and when actively breeding on the egg-food supplemented seed diet.
These findings suggest that these factors did not influence the VLDL particle diameter distribution parameters measured.

\section{Estimating VLDLy particle diameter range in zebra finches}

Previous studies on egg production in domestic fowl have found that VLDL particles of different diameters vary in the extent to which they contribute to yolk formation, and consequently in their ability to support egg production (Perry and Gilbert, 1979; Griffin and Perry, 1985; Griffin and Hermier, 1988; Walzem et al., 1994; Walzem, 1996; Walzem et al., 1999). Basing VLDLy particle diameter range on the proposed 'sieving' properties of the ovarian granulosa basal laminae of laying chickens and turkeys provided a comparable estimate of VLDLy particle diameter for zebra finches and chickens, as the proportion of VLDL particles from non-laying females that fell within this range was minimal in both species (38\% in zebra finches and 19\% in chickens). The majority of VLDL particles of laying chickens $(61 \%)$ and half of the particles of laying zebra finches fell within the SVLDLy range. This suggests that similarities exist in the sieving properties of the ovaries of different species of birds. Future studies are required to compare the composition, structure and sieving properties of the ovarian granulosa basal laminae of domesticated and non-domesticated birds to determine whether ovarian sieving of VLDL particles influences selection acting on VLDL particle diameter.

Basing the VLDLy range on Walzem's original correlation method (Walzem, 1996) also resulted in comparable estimates of VLDLy particle diameter for zebra finches and chickens, as the majority of VLDL particles from laying females fell within the cVLDLy range (63\% in zebra finches; $70 \%$ in chickens). However, the majority of VLDL particles of non-laying zebra finches also fell within this range (51\%, compared with only $25 \%$ of VLDL particles of non-laying chickens). Our modified correlation method based on data from laying zebra finches resulted in a diameter range that encompassed a majority of VLDL particles from non-laying zebra finches (59\%, cf. $25 \%$ of VLDL particles from non-laying chickens within the cVLDLy range). Moreover, the zVLDLy range encompassed only $40 \%$ of VLDL particles from laying females (cf. $70 \%$ of laying chicken VLDL particles within the cVLDLy range). The discrepancies between the proportion of VLDL particles that fell within the cVLDLy in chickens and the zVLDLy in zebra finches may be due to differences in the way the ranges were calculated. Many of the VLDL particle diameter classes that were positively associated with laying rate in chickens, and therefore made up the cVLDLy range, had statistically significant correlation coefficients $(P<0.05$ for $r$-values greater than 0.444) (Walzem, 1996). By contrast, all of the VLDL particle diameter classes that were positively correlated with residual mean egg mass in zebra finches, and therefore made up the zVLDLy range, did not have statistically significant correlation coefficients $(P>0.1$ in all cases). Consequently, the zVLDLy range appears to be the least reliable estimate of VLDLy particle diameter range in zebra finches. 


\section{Metabolic shifts in VLDL particle diameter distribution}

As predicted, female zebra finches exhibited less dramatic shifts in lipid metabolism during egg production. However, this was mainly due to the unexpected finding that the majority of VLDL particles of non-laying zebra finches were very small in diameter $(57 \%$ of particles had diameters less than $30 \mathrm{~nm}$ ). Consequently, the diameter distributions of laying zebra finches actually shifted towards larger VLDL particles compared to the distributions of non-laying females. Furthermore, the diameter distributions of both laying and non-laying zebra finches peaked at small VLDL particles, and therefore overlapped considerably. Similar results have been reported for comparisons between growing (i.e. immature) and egg-producing Tsaiya ducks, Anas platyrhynchos domestica (Lien et al., 2005). When provided with ad libitum access to food, domesticated Tsaiya ducks had VLDL particle diameter distributions, as assessed by transmission electron microscopy, that included more larger particles (range: $50-75 \mathrm{~nm}$ ) and had larger mean VLDL particle diameters during egg production at 30 weeks of age $(61.57 \pm 1.98 \mathrm{~nm})$ than while actively growing at 12 weeks of age (range: $35-60 \mathrm{~nm}$; mean diameter: $47.67 \pm 2.37 \mathrm{~nm}$ ) (Lien et al., 2005). This is in stark contrast to the data from chickens, wherein less than $10 \%$ of the VLDL particles measured at the peak of egg laying had diameters larger than $51 \mathrm{~nm}$ (cf. nearly $60 \%$ of particles in nonlaying chickens), resulting in very little overlap between the VLDL distributions of laying and non-laying chickens.

Laying zebra finches, like laying chickens, had higher circulating triacylglyceride levels and more particles within the sVLDLy and cVLDLy ranges than non-laying females, despite the fact that laying zebra finches had fewer very small VLDL particles, more large VLDL particles, and wider diameter distributions than non-laying females. Furthermore, the VLDL particle diameter distributions of zebra finches and chickens shifted towards similar modal and median VLDL particle diameters during egg production. These results suggest that, regardless of the direction that VLDL particle diameter distributions have to shift, specific changes in lipid metabolism (e.g. increased lipid production and maintenance of a large proportion of small VLDL particles of specific diameters) may be essential for egg production in both domesticated and nondomesticated birds. However, data on reproductive status and VLDL particle diameter distribution from more domesticated and free-living avian species are required to confirm the relationship between changes in lipid metabolism and avian egg production.

The differences in VLDL particle diameter distribution between non-laying chickens and zebra finches observed in this study may be due to differences in rates of lipid turnover due to variation in metabolic rate. Based on allometric scaling of metabolic rate (for reviews, see Calder, 1981; Taylor, 1987), smaller passerine songbirds have higher mass-specific metabolic rates than larger chickens (Lasiewski and Dawson, 1967; Reynolds and Lee, 1996; McKechnie and Wolf, 2004). Consequently, passerine songbirds also have higher rates of lipid turnover. When VLDL particles undergo lipoprotein lipasemediated metabolism, triacylglycerol is removed by hydrolysis, and surface lipids and apolipoproteins (e.g. apo-A, apo-C) are transferred to other lipoprotein particles (e.g. high density lipoproteins) (for reviews, see Eisenberg, 1986; Walzem, 1996). This results in a decrease in VLDL particle size and an increase in particle density, and the eventual conversion to intermediate density lipoproteins (IDL) and then low density lipoproteins (for reviews, see Eisenberg, 1986; Walzem, 1996). Hermier et al. (Hermier et al., 1985) reported that IDL particles from immature chickens had an average diameter of $20.0 \mathrm{~nm}$. Therefore, the abundance of very small VLDL particles observed in non-laying zebra finches $(57 \%$ under $30 \mathrm{~nm}$ in diameter, cf. $<1 \%$ in nonlaying chickens) may have actually been IDL particles resulting from the rapid metabolism of larger VLDL particles.

Differences in the environmental conditions that chickens and zebra finches are exposed to during egg production may also influence the proportion of large VLDL particles in circulation. Domestic chickens are generally housed under conditions that promote optimal egg production, e.g. lightcontrolled facilities, a diet regime tailored for egg production, vaccinations against disease, and husbandry practices that eliminate parasites (Etches, 1996). Consequently, they are capable of meeting their own metabolic needs via hydrolysis of the small VLDLy particles (Bacon and Musser, 1977; Bacon et al., 1978; Bacon, 1981) and possibly renal generic VLDL (Walzem et al., 1999) despite the increased resistance of VLDLy to hydrolysis by lipoprotein lipase (Bacon et al., 1978; Bacon, 1981; Griffin et al., 1982; Hermier et al., 1989; Schneider et al., 1990; Walzem et al., 1994; Walzem, 1996). Alternatively, previous studies on laying chickens have suggested that individual hepatocytes may vary in their functional capacity to initiate apoVLDL-II, and thus VLDLy, synthesis in response to elevated levels of oestrogen (Lin and Chan, 1981; Lin et al., 1986). Consequently, it is possible that the livers of laying chickens continue to make small amounts of larger, generic VLDL in sufficient quantities to meet the laying females' energetic requirements. The rapid and continuous metabolism of such generic VLDL by highly productive layers would leave minimal concentrations in circulation relative to VLDLy, making their ready detection in laying domestic fowl challenging. By contrast, reproduction in free-living, non-domesticated birds is generally timed to ensure that the period of chick rearing coincides with the period of peak food abundance (Perrins, 1970). Therefore, egg production in these birds often occurs during periods of lower food availability and unpredictable environmental conditions earlier in the breeding season (Williams, 1998). Maintenance of large VLDL particles in circulation may act to buffer the fluctuating, environmentally dependent energetic demands of these laying birds. The extent to which hepatic VLDLy and renal generic VLDL contribute to the levels of utilizable VLDL present in laying, non-gallinaceous birds, and in particular, in free-living birds faced with far less predictable conditions, remains unknown. Future studies are needed to assess whether the larger VLDL particles observed in laying zebra finches contain apoVLDL-II, in order to determine whether these particles are generic or yolk-targeted VLDL. 
In contrast to domesticated birds that have undergone directional selection for specific traits, such as continuous egg production or rapid growth, the selective pressures on laying zebra finches, and on non-domesticated birds in general, are generally focused on maintaining traits that maximize the trade-off between current reproductive effort and future fecundity and survival (Williams, 1966; Stearns, 1992; Bernardo, 1996). The increased LPL-resistance of VLDLy may result in selection for the maintenance of larger, potentially generic, VLDL particles in non-domesticated birds during egg production, as observed in zebra finches in this study (19\%) and Tsaiya ducks $(\sim 100 \%)$ (Lien et al., 2005), to ensure that laying females have an ample supply of VLDL that can be metabolized in case their own energetic demands increase during egg production due to changes in environmental conditions. Data on VLDL particle diameter distribution during egg production in many more free-living avian species, including other gallinaceous and passerine birds, are needed to determine whether the differences between chickens and zebra finches observed in this study are, in fact, due to differences in selective pressures on these birds, or to phylogenetic differences that are unrelated to inter-specific differences in adaptations to egg production.

In addition to egg production, reproduction in nondomesticated species generally involves broody behaviour, i.e. incubation and post-hatching parental care (e.g. provisioning and brooding of young). This is in contrast to many breeds of domesticated chickens, whose reproductive activity is limited to egg production as a result of commercial practices (e.g. photoperiod manipulation, egg removal) and decreases in broodiness, hatchability and fertility as a consequence of selection for increased egg production (Emmerson et al., 1991; Nestor et al., 1996; Sewalem et al., 1998) (reviewed by Romanov, 2001). Therefore, given that non-domesticated, laying females must ensure that they have adequate resources to perform post-laying parental behaviours, they may limit lipid allocation to current egg production in exchange for allocating more energy towards self-maintenance (i.e. maintaining larger VLDL particles) to enhance their chances for survival through the current reproductive period and beyond, thus maximizing current and potentially future reproductive effort. Further studies are needed that will assess the relationships between variation in VLDL particle diameter distribution during egg production in free-living avian species and both current and future reproductive success, and maternal survival and longevity.

\section{List of abbreviations}

$\begin{array}{ll}\text { VLDL } & \text { very low density lipoprotein } \\ \text { VLDLy } & \text { yolk-targeted VLDL } \\ \text { VTG } & \text { vitellogenin } \\ \text { LPL } & \text { lipoprotein lipase } \\ \text { cVLDLy } & \text { chicken VLDLy } \\ \text { sVLDLy } & \text { avian-ovary sieved VLDLy } \\ \text { zVLDLy } & \text { zebra finch VLDLy }\end{array}$

This study was funded by a Natural Sciences and Engineering Research Council of Canada Operating Grant to T.D.W., a Natural Sciences and Engineering Research Council of Canada Post-Graduate Scholarship to K.G.S. and project 8736 of the Texas Agricultural Experiment Station to R.L.W. We would like to thank Kendall Hood, Mikhael Wallowitz and Wene Yan for their assistance with the VLDL particle diameter assay, and Miriam Ben Hamida, Mathilde Curnillon, Gina Eom and Pamela Smith for their help with the triacylglyceride assay and seed consumption data collection.

\section{References}

Bacon, W. L. (1981). Metabolism of lipid labeled very low density lipoprotein from laying turkey hens in laying turkey hens and immature turkeys. Poult. Sci. 60, 1525-1536.

Bacon, W. L. and Musser, M. A. (1977). The turnover rate of lipoprotein of $\mathrm{d}<1.006$ from plasma of laying turkey hens. Poult. Sci. 56, 35-41.

Bacon, W. L., Musser, M. A. and Brown, K. I. (1974). Plasma free fatty acid and neutral lipid concentration in immature, laying and broody turkey hens. Poult. Sci. 53, 1154-1160.

Bacon, W. L., Leclercq, B. and Blum, J. C. (1978). Difference in metabolism of very low density lipoprotein from laying chicken hens in comparison to immature chicken hens. Poult. Sci. 57, 1675-1686.

Bergink, E. W., Wallace, R. A., Van de Berg, J. A., Bos, E. S., Gruber, M. and Geert, A. B. (1974). Estrogen-induced synthesis of yolk proteins in roosters. Am. Zool. 14, 1177-1193.

Bernardo, J. (1996). The particular maternal effect of propagule size, especially egg size: patterns, models, quality of evidence and interpretations. Am. Zool. 36, 216-236.

Blue, M.-L., Protter, A. A. and Williams, D. L. (1980). Biosynthesis of apolipoprotein B in rooster kidney, intestine, and liver. J. Biol. Chem. 255, 10048-10051.

Boyle-Roden, E. and Walzem, R. L. (2005). Integral apolipoproteins increase surface-located triacylglycerol in intact native apo-protein $\mathrm{B}_{100}$ containing lipoproteins. J. Lipid Res. 46, 1624-1632.

Calder, W. A. (1981). Scaling of physiological processes in homeothermic animals. Annu. Rev. Physiol. 43, 301-322.

Challenger, W. O., Williams, T. D., Christians, J. K. and Vezina, F. (2001). Follicular development and plasma yolk precursor dynamics through the laying cycle in the European starling (Sturnus vulgaris). Physiol. Biochem. Zool. 74, 356-365.

Chan, L., Jackson, R. L., O'Malley, B. W. and Means, A. R. (1976). Synthesis of very low density lipoproteins in the cockerel. J. Clin. Invest. 58, 368-379.

Chapman, M. J. (1980). Animal lipoproteins: chemistry, structure, and comparative aspects. J. Lipid Res. 21, 789-853.

Chen, S.-E., Long, D. W., Nestor, K. E., Walzem, R. L., Meuniot, V. L., Zhu, H., Hansen, R. J. and Bacon, W. L. (1999). Effect of divergent selection for total plasma phosphorus on plasma and yolk very low density lipoproteins and plasma concentrations of selected hormones in laying Japanese quail. Poult. Sci. 78, 1241-1251.

Christians, J. K. and Williams, T. D. (1999). Organ mass dynamics in relation to yolk precursor production and egg formation in European Starlings Sturnus vulgaris. Physiol. Biochem. Zool. 72, 455-461.

Dashti, N., Kelley, J. L., Thayer, R. H. and Ontko, J. A. (1983). Concurrent inductions of avian hepatic lipogenesis, plasma lipids, and plasma apolipoprotein B by estrogen. J. Lipid Res. 24, 368-380.

Deeley, R. G., Mullinix, K. P., Wetekam, W., Kronenberg, H. M., Meyers, M., Eldridge, J. D. and Goldberger, R. F. (1975). Vitellogenin synthesis in the avian liver. J. Biol. Chem. 250, 9060-9066.

Eisenberg, S. (1986). Plasma lipoprotein conversions. Meth. Enzymol. 129, 347-366.

Emmerson, D. A., Anthony, N. B. and Nestor, K. E. (1991). Genetics of growth and reproduction in the turkey. 11. Evidence of nonadditive genetic variation. Poult. Sci. 70, 1084-1091.

Etches, R. J. (1996). Reproduction in Poultry. Wallingford: CAB International.

Griffin, H. D. (1981). Plasma very low density lipoproteins in immature and laying hens Gallus domesticus. Biochem. Soc. Trans. 9, 115P. 
Griffin, H. and Hermier, D. (1988). Plasma lipoprotein metabolism and fattening in poultry. In Leanness in Domestic Birds: Genetic, Metabolic and Hormonal Aspects (ed. B. Leclercq and C. C. Whitehead), pp. 175-201. New York: Butterworth.

Griffin, H. D. and Perry, M. M. (1985). Exclusion of plasma lipoproteins of intestinal origin from avian egg yolk because of their size. Comp. Biochem. Physiol. 82B, 321-325.

Griffin, H., Grant, G. and Perry, M. (1982). Hydrolysis of plasma triacylglycerol-rich lipoproteins from immature and laying hens (Gallus domesticus) by lipoprotein lipase in vitro. Biochem. J. 206, 647-654.

Gruber, M. (1972). Hormonal control of yolk protein synthesis. In Egg Formation and Production (ed. B. M. Freeman and P. E. Lake), pp. 23-34. Edinburgh: British Poultry Science.

Hermier, D., Forgez, P. and Chapman, M. J. (1985). A density gradient study of the lipoprotein and apolipoprotein distribtion in the chicken, Gallus domesticus. Biochim. Biophys. Acta 836, 105-118.

Hermier, D., Forgez, P., Williams, J. and Chapman, M. J. (1989). Alterations in plasma lipoproteins and apolipoproteins associated with estrogen-induced hyperlipidemia in the laying hen. Eur. J. Biochem. 184, 109-118.

Kudzma, D. J., Swaney, J. B. and Ellis, E. N. (1979). Effects of estrogen administration on the lipoproteins and apoproteins of the chicken. Biochim. Biophys. Acta 572, 257-268.

Lasiewski, R. C. and Dawson, W. R. (1967). A re-examination of the relation between standard metabolic rate and body weight in birds. Condor 66, 477490.

Lien, T. F., Jan, D. F. and Chen, K. L. (2005). Lipoprotein profiles and components in Tsaiya ducks under ad libitum feeding and fasting. Comp. Biochem. Physiol. 142A, 325-330.

Lin, C. T. and Chan, L. (1981). Estrogen regulation of yolk and non-yolk protein synthesis in the avian liver. An immunocytochemical study. Differentiation 18, 105-114.

Lin, C. T., Palmer, W., Wu, J. Y. and Chan, L. (1986). Estrogen induction of very low density apolipoprotein II synthesis, a major avian liver yolk protein, involves the recruitment of hepatocytes. Endocrinology 118, 538544.

McKechnie, A. E. and Wolf, B. O. (2004). The allometry of avian basal metabolic rate: good predictions need good data. Physiol. Biochem. Zool. 77, 502-521.

Mitchell, M. A. and Carlisle, A. J. (1991). Plasma zinc as an index of vitellogenin production and reproductive status in the domestic fowl. Comp. Biochem. Physiol. 100A, 719-724.

National Research Council Subcommittee on Poultry Nutrition (1994). Nutrient Requirements of Poultry (9th edn). Washington, DC: National Academy Press.

Neilson, J. T. M. and Simpson, C. F. (1973). Plasma lipoproteins of turkeys injected with a single dose of diethylstilbesterol. Atherosclerosis 18, 445450.

Nestor, K. E., Noble, D. O., Zhu, J. and Moritsu, Y. (1996). Direct and correlated responses to long-term selection for increased body weight and egg production in turkeys. Poult. Sci. 75, 1180-1191.

Peebles, E. D., Burnham, M. R., Walzem, R. L., Branton, S. L. and Gerard, P. D. (2004). Effects of fasting on serum lipids and lipoprotein profiles in the egg-laying hen (Gallus domesticus). Comp. Biochem. Physiol. 138A, 305-311.

Perrins, C. M. (1970). The timing of birds' breeding seasons. Ibis 112, 242255.

Perry, M. M. and Gilbert, A. B. (1979). Yolk transport in the ovarian follicle of the hen (Gallus domesticus): lipoprotein-like particles at the periphery of the oocyte in the rapid growth phase. J. Cell Sci. 39, 257272.
Romanov, M. N. (2001). Genetics of broodiness in poultry - a review. Asianaustralas J. Anim. Sci. 14, 1647-1654.

Reynolds, P. S. and Lee, R. M. (1996). Phylogenetic analysis of avian energetics: passerines and non-passerines do not differ. Am. Nat. 147, 735759.

Salvante, K. G. and Williams, T. D. (2002). Vitellogenin dynamics during egg-laying: daily variation, repeatability and relationship with egg size. $J$. Avian Biol. 33, 391-398.

SAS Institute (1999). SAS OnlineDoc, Version 8. Cary: SAS Institute.

Schneider, W. J., Carroll, R., Severson, D. L. and Nimpf, J. (1990). Apolipoprotein VLDL-II inhibits lipolysis of triacylglyceride-rich lipoproteins in the laying hen. J. Lipid Res. 31, 507-513.

Sewalem, A., Johansson, K., Carlgren, A.-B., Wilhelmson, M. and Lillpers, K. (1998). Are reproductive traits impaired by selection for egg production in hens? J. Anim. Breed. Genet. 115, 281-297.

Speake, B. K., Murray, A. M. B. and Noble, R. C. (1998). Transport and transformations of yolk lipids during development of the avian embryo. Prog. Lipid Res. 37, 1-32.

Stearns, S. C. (1992). The Evolution of Life Histories. Oxford: Oxford University Press.

Tarugi, P., Ballarini, G., Pinotti, B., Franchini, A., Ottaviani, E. and Calandra, S. (1998). Secretion of apoB- and apoA-I-containing lipoproteins by chick kidney. J. Lipid Res. 39, 731-743.

Taylor, C. R. (1987). Structural and functional limits to oxidative metabolism: insights from scaling. Anпu. Rev. Physiol. 49, 135-146.

Véniant, M. M., Sullivan, M. A., Kim, S. K., Ambroziak, P., Chu, A., Wilson, M. D., Hellerstein, M. K., Rudel, L. L., Walzem, R. L. and Young, S. G. (2000). Defining the atherogenicity of large and small lipoproteins containing apolipoprotein B100. J. Clin. Invest. 106, 1501-1510.

Wallace, R. A. (1985). Vitellogenisis and oocyte growth in non-mammalian vertebrates. In Developmental Biology, Vol. 1, Oogenesis (ed. L. W. Browder), pp. 127-177. New York: Plenum Press.

Walzem, R. L. (1996). Lipoproteins and the laying hen: form follows function. Poult. Avian Biol. Rev. 7, 31-64.

Walzem, R. L., Davis, P. A. and Hansen, R. J. (1994). Overfeeding increases very low density lipoprotein diameter and causes the appearance of a unique lipoprotein particle in association with failed yolk deposition. J. Lipid Res. 35, 1354-1366.

Walzem, R. L., Hansen, R. J., Williams, D. L. and Hamilton, R. L. (1999). Estrogen induction of VLDLy assembly in egg-laying hens. J. Nutr. 129, $467 \mathrm{~S}-472 \mathrm{~S}$.

Williams, G. C. (1966). Adaptation and Natural Selection. Princeton: Princeton University Press.

Williams, T. D. (1996). Variation in reproductive effort in female Zebra Finches (Taeniopygia guttata) in relation to nutrient-specific dietary supplements during egg laying. Physiol. Zool. 69, 1255-1275.

Williams, T. D. (1998). Avian reproduction, overview. In Encyclopedia of Reproduction. Vol. 1 (ed. E. Knobil and J. D. Neil), pp. 325-336. New York: Academic Press.

Williams, T. D. and Christians, J. K. (1997). Female reproductive effort and individual variation: neglected topics in environmental endocrinology? In Proceedings of the 13th International Congress of Comparative Endocrinology (ed. S. Kawashima and S. Kikuyama), pp. 1669-1675. Yokohama: Monduzzi Editore.

Williams, T. D. and Martyniuk, C. (2000). Tissue mass dynamics during eggproduction in female Zebra Finches, Taeniopygia guttata: dietary and hormonal manipulations. J. Avian Biol. 31, 87-95.

Williams, T. D. and Miller, M. (2003). Individual and resource-dependent variation in ability to lay supranormal clutches in response to egg removal. Auk 120, 481-489.

Zar, J. H. (1996). Biostatistical Analysis. London: Prentice Hall. 